Copyright ( 2015 IEEE. Personal use of this material is permitted. Permission from IEEE must be obtained for all other uses, in any current or future media, including reprinting/republishing this material for advertising or promotional purposes, creating new collective works, for resale or redistribution to servers or lists, or reuse of any copyrighted component of this work in other works. 


\section{Design and Experiment of Frequency Offset Estimation and Compensation in High-speed Underwater Acoustic Communication}

\author{
Yunqiang Bi, Zhiqiang He, Weipeng Jiang, and Kai Niu \\ Key Lab of Universal Wireless Communications, \\ Ministry of Education \\ Beijing University of Posts and Telecommunications, \\ Beijing China
}

\author{
Yue Rong \\ Department of Electrical and Computer Engineering \\ Department of Imaging and Applied Phisics \\ Curtin University, Bently, WA, Australia
}

\begin{abstract}
In underwater acoustic (UWA) communication, Doppler effect is particularly severe due to the slow velocity of sound and the complex variant UWA channel environment. Carrier frequency offset (CFO) can result in extension and compression of the received signal in time domain and has a direct effect on the performance of decoding. In this paper, we propose a new scheme of CFO estimation and compensation for a high speed UWA communication system. There are three steps including coarse CFO estimation, fine CFO estimation and linear interpolation, which are taken to estimate and compensate the CFO. The scheme can eliminate the phenomenon of ambiguous phase and tolerate quick random variation of the CFO in UWA channel. A UWA communication experiment was carried out in December 2012 in the Indian Ocean, off Rottnest Island, Western Australia. With the proposed algorithm in this paper, the UWA system can achieve an average of $1.95 \%$ uncoded BER with QPSK modulation at the $1 \mathrm{~km}$ range and $5.57 \%$ with $B P S K$ at the $10 \mathrm{~km}$ range.
\end{abstract}

Keywords-Underwater acoustic communication, carrier frequency offset, estimation and compensation, experiment

\section{INTRODUCTION}

The UWA channel is affected by many factors and is much more complicated than wireless channel in the air. In fact, UWA communication turns out to be particularly challenging due to the extremely limited bandwidth, large Doppler shifts, severe channel fading, and strong multipath interference $[1,2]$.

Sound is chosen as the favorite transmission medium for this specific scenario due to its superior propagation characteristics. However, the system's performance is easy to be affected by carrier frequency offset (CFO) because of the slow speed of sound in water $(\mathrm{c}=1500 \mathrm{~m} / \mathrm{s})$ [3]. Even small motion between the transmitter and receiver can cause severe Doppler effect. What's more, the carrier frequency can be easily affected by many unpredictable factors of sea environment, such as temperature, salinity, depth, storms and ocean currents, which result in the random variation of CFO. Doppler can expand or compress the transmitted signal in time domain, interfere with the carrier frequency tracking and symbol synchronization and finally increase the error rate.

This work is supported by National High Technology Research and Development Program of China (863 Program SS2015AA011303), National Natural Science Foundation of China (61171099), and the Australian Research Council's Discovery Projects funding scheme (DP140102131).
Usually traditional methods get the CFO by setting pilot at the zero point of the signal spectrum [4]. The CFO is calculated in frequency domain and is compensated by adjusting the sampling rates. This method will consume a lot of power to transmit pilot. Another way is to add a small piece of synchronous head in the front of each frame and obtain the CFO by matched filter or FFT transform [5]. Both methods will perform badly when the CFO is relatively large and swings sharply. Recent years, some methods of CFO estimation and compensation for orthogonal frequency division multiplexing (OFDM) are proposed [6-8]. These algorithms get some improvement with the cost of a certain level of complexity. However, OFDM is sensitive to peak-to-average power ratio (PAPR) and CFO, and has high linearity requirements for the preamp of UWA equipment [9]. Single-carrier frequency-domain equalization (SC-FDE) [10] offers an alternative choice to achieve similar performance and processing complexity but without the drawbacks. In this paper, we propose a new practical CFO estimation and compensation scheme for a UWA system based on SC-FDE. The performance was verified by a sea experiment conducted in December 2012 in the Indian Ocean off Rottnest Island, Western Australia.

Three steps are adopted to obtain the CFO, including coarse frequency offset estimation, fine frequency offset estimation and linear interpolation. Received data are compensated by resampling with the $\mathrm{CFO}$ estimated. Then we conduct the channel estimation based on compressed sensing [11], iterative frequency-domain equalization with soft decision feedback [12] and channel decoding. In the experiment, there was one transmitting transducer and one receiving hydrophone. The transmitting transducer was drifted with an average speed of $0.96 \mathrm{~m} / \mathrm{s}$. The peak drift speed was $1.7 \mathrm{~m} / \mathrm{s}$. With the proposed algorithm in this paper, the UWA system can achieve an average of $1.95 \%$ uncoded BER with QPSK modulation over a range of $1 \mathrm{~km}$ and $5.57 \%$ uncoded BER with BPSK over a range of $10 \mathrm{~km}$.

The rest of the paper is organized as follows. Section II gives the signal model. Section III focuses on the introduction of the CFO estimation and compensation scheme. In Section IV and $\mathrm{V}$ we present the detailed experiment conditions and results. Section VI concludes the paper. 


\section{SySTEM MOdEL}

In this section, we establish mathematical models for the system. This paper just consider a single-input-single-output (SISO) scenario with one user.

Fig. 1 shows the block diagram of our UWA communication system. The information bits $b(t)$ are mapped into symbols after channel coding. With the reference symbols, which are used for synchronization and channel estimation, they compose the data $d_{k}$ to be transmitted. As shown in Fig. 2, each data frame has $N$ symbols including $\left(N-N_{c p}\right)$ information symbols and $N_{c p}$ training symbols. After carrier modulation, the transmitted data $x(t)$ passes through the underwater channel and is received as $y(t)$. At the receiver, synchronization, CFO estimation and compensation by resampling will be conducted first, followed with the channel estimation, iterative equalization in frequency-domain and channel decoding.

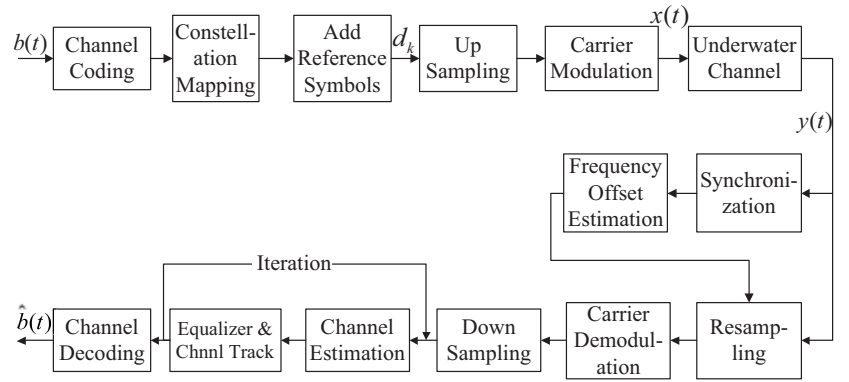

Fig. 1. Block diagram of the UWA communication system

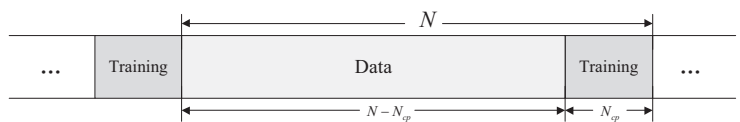

Fig. 2. Data frame structure

The transmitted signal can be written as

$$
x(t)=\operatorname{Re}\left\{d_{k} g(t) e^{j 2 \pi f_{c} t}\right\}
$$

where $d_{k}(k=1,2 \ldots)$ are the transmitted symbols modulated including the synchronous reference symbols. $g(t)$ is the transmitting filter of raised cosine function . Re $\{$.$\} denotes$ the real part. $f_{c}$ is the carrier frequency. Consider a UWA channel where the number of multipath is $L$, and each path is assumed to share a common Doppler scaling factor with a different time delay. So the received signal can be expressed as

$$
y(t)=\sum_{l=0}^{L-1} h_{l} x\left((1+\alpha) t-\tau_{l}\right)+n(t)
$$

where $h_{l}$ is the path gain and $\tau_{l}$ is the time delay of the $l$-th multipath. $n(t)$ is additive white Gaussian noise (AWGN). The Doppler scaling factor $\alpha=v / v_{s}$, where $v_{s}$ is the speed of sound in water and $v$ is the speed of moving transmitter in the direction of acoustic wave propagation. Based on (1), we can write the received signal as

$$
y(t)=\operatorname{Re}\left\{\sum_{l=0}^{L-1} h_{l} d_{k} g\left((1+\alpha) t-\tau_{l}\right) e^{j 2 \pi f_{c}\left((1+\alpha) t-\tau_{l}\right)}\right\}+n(t)
$$

To eliminate the Doppler effect and ignoring the multipath delay $\tau_{l}$, we resample the data with sampling interval $T_{s}^{\prime}=$ $T_{s} /(1+\alpha)$, where $T_{s}$ is the original symbol duration. The resampled data $r(n)=y\left(n T_{s}^{\prime}\right)$ is obtained as

$$
\begin{aligned}
r(n) & =\operatorname{Re}\left\{\sum_{l=0}^{L-1} h_{l} d_{k} g\left((1+\alpha) n T_{s}^{\prime}-\tau_{l}\right) e^{j 2 \pi f_{c}\left((1+\alpha) n T_{s}^{\prime}-\tau_{l}\right)}\right\} \\
& +n\left(n T_{s}^{\prime}\right)
\end{aligned}
$$

The Doppler shift $f_{d}$ can be expressed as

$$
f_{d}=\alpha f_{c}=\left(\frac{T_{s}}{T_{s}^{\prime}}-1\right) f_{c}
$$

The Doppler phase $\varphi_{d}$ can be expressed as

$$
\varphi_{d}=2 \pi n T_{s} f_{d}
$$

\section{FREQUENCY OFFSET ESTIMATION AND COMPENSATION}

Fig. 3 shows the block diagram of the CFO estimation and compensation scheme we proposed. The received data are discretized by the original sampling interval $T_{s}$ first. Then we conduct the synchronization to confirm the frame head and begin the coarse CFO estimation. The coarse CFO estimation gives a rough estimation of the CFO $f_{d}^{\prime n}$ while a more precise value $f_{d}^{n}$ is obtained by the fine CFO estimation next. Then $\mathrm{CFO}$ of current and previous frames are utilized to get a group of Doppler shift values by linear interpolation to track the channel changes within a frame, with which we resample the received data to compensate the $\mathrm{CFO}$.

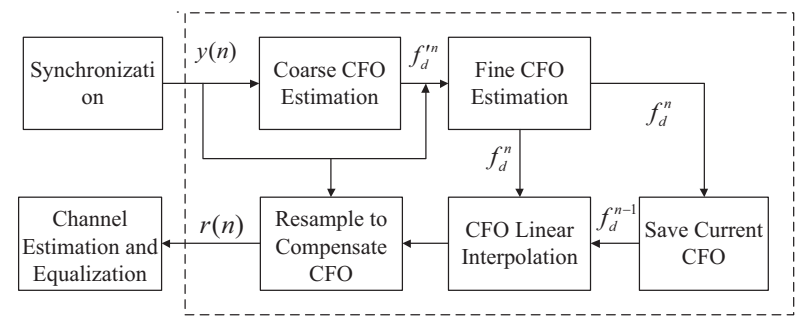

Fig. 3. Procedure of $\mathrm{CFO}$ estimation and compensation

\section{A. Coarse frequency offset estimation}

In this section, a result of coarse frequency offset estimation is figured out. As shown in Fig. 4, the $N_{c p}$ length training sequence is divided into $G$ groups and a tail, $G=$ floor $\left(N_{c p} / S\right)$ and each group has $S$ samples. The $\left(N_{c p}-G *\right.$ $S$ ) length tail is abandoned. $S$ is even. First a correlation is

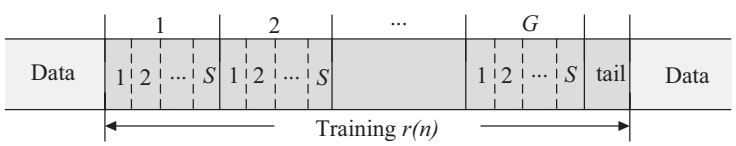

Fig. 4. Training structure of coarse CFO estimation 
done between the received training sequence $r(n)$ and local training sequence $c(n)$

$$
\begin{aligned}
r^{\prime}(n)=r^{\prime} & {[(q-1) S+p]=} \\
& \frac{r[(q-1) S+p] c^{*}[(q-1) S+p]}{\sqrt{\frac{1}{S} \sum_{k=1}^{S} r[(q-1) S+p] r^{*}[(q-1) S+p]}}
\end{aligned}
$$

where $p=1,2 \cdots S, q=1,2 \cdots G$ and an energy normalization process is done for samples of each group. Therefor we can express the Doppler phase $\varphi_{d}^{\prime n}$ during the time of $S / 2$ symbols period $S T_{s} / 2$ as

$$
\varphi_{d}^{\prime n}=\arg \left(\frac{2}{G S} \sum_{i=1}^{G} \sum_{j=1}^{S / 2} r^{\prime}[(i-1) S+j] r^{\prime *}\left[(i-1) S+j+\frac{S}{2}\right]\right)
$$

Thus, the result of coarse CFO estimation can be represented as

$$
f_{d}^{\prime n}=\frac{\varphi_{d}^{\prime n}}{\pi S T_{s}}
$$

Here a proper length of $S$ must be chosen to avoid a phase ambiguous phenomenon, especially in high-speed UWA scenario. As shown in Fig. 5, there is a zigzag relationship between the Doppler phase $\varphi_{d}^{\prime n}$ and Doppler shift $f_{d}^{\prime n}$. And the relation (10) holds below:

$$
\varphi_{d}^{\prime n}=\pi f_{d}^{\prime n} T_{s} S \in\left[-\frac{\pi}{2}, \frac{\pi}{2}\right], f_{d}^{\prime n} \in\left[-\frac{1}{2 S T_{s}}, \frac{1}{2 S T_{S}}\right]
$$

When given a phase we can only confirm the Doppler shift in the interval $\left[-1 / 2 S T_{s}, 1 / 2 S T_{s}\right]$ according to (9). In highspeed moving scenario, when the Doppler is large and outside the interval, the phase will be ambiguous. However we can choose a smaller $S$ to get a bigger interval range to cover the Doppler. That is why we divide the training sequence into groups to perform the coarse CFO estimation. Through this method, the system can accommodate the UWA channel where the Doppler is relatively large or swings sharply. But smaller $S$ may result in the decline of the estimation's precision. Therefore we should choose a proper $S$ to not only cover the Doppler in the effective interval but also keep a certain accuracy according to the specific UWA channel environment.

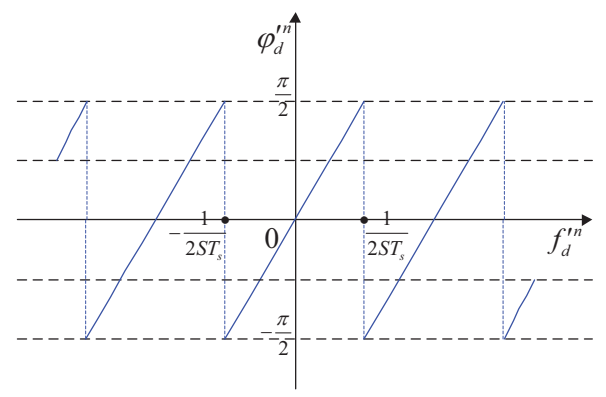

Fig. 5. Relationship of CFO phase and Doppler

\section{B. Fine frequency offset estimation}

In this subsection, we will give a more accurate value by bisection method based on the result of coarse CFO estimation.
Fig. 6 shows the basic process of the algorithm. Fig. 7 gives the schematic diagram.

An small initial iteration interval $\left[f_{0}-\Delta, f_{0}+\Delta\right]$ is set in Step0. $f_{0}$ is initialized by $f_{d}^{\prime n}$ produced in section A. The variable flag denotes the residual CFO phase of the training after resampling in each round of iteration. Received data $y(n)$ is resampled by the new sampling rate calculated by $f_{\text {mid }}$ in Step1 and is resynchronized to find the new frame head in Step2. The residual offset phase of the resampled data after the CFO compensation in current iteration is calculated in Step3 by the method in section A, but with a bigger $S$ to guarantee a more accurate result. In Step4, new interval will be decided by the sign of the residual offset phase. If the phase has reached the judgment threshold $\xi$, the algorithm stops. If not, new iteration will begin from Step1.

Fine CFO Estimation Algorithm
Step0 : $f_{\text {low }}=f_{0}-\Delta, f_{\text {high }}=f_{0}+\Delta, f_{\text {mid }}=\left(f_{\text {low }}+f_{\text {high }}\right) / 2$, flag $=2 \pi T_{s} f_{0}$
Step1 : Resample the received data $y(n)$ by the sample rate $f_{s}\left(1+f_{\text {mid }} /\right.$
$\left.f_{c}\right), f_{s}$ is the original sample rate, $f_{c}$ is the carrier frequency;
Step2 : Resynchronize the resampled data and confirm the data frame
head;
Step3 : Calculate the frequency offset phase flag by the method of co-
arse frequency offset;
Step4: If $\mid$ flag $\mid<\xi, f_{\text {mid }}$ is the fine frequency offset $f_{d}^{n}$ we want, else,
if flag $>0, f_{\text {high }}=f_{\text {mid }}$, if flag $\leq 0, f_{\text {low }}=f_{\text {mid }}$, and turn to Step1.

Fig. 6. Fine $\mathrm{CFO}$ estimation algorithm

To ensure the success of the algorithm, $S$ in coarse CFO estimation must be appropriate to obtain the right Doppler range. And the setting of the value $\Delta$ must make sure that the initialized interval in Step0 covers the final result but is not outside the sawtooth wave period above. In fact, in the experiment of $1 \mathrm{~km}$ communication scenario where the Doppler shift wasn't so severe, $S$ was set as 200 in coarse CFO estimation while 500 in this section. $\Delta$ was set as $1.25 \mathrm{~Hz}$ and $\xi$ was set as $10^{-4}$. It is need to be noticed that the algorithm will iterate towards the actual CFO by the complexity of $O(\log n)$, but will not improve much when $\xi$ is set too small.

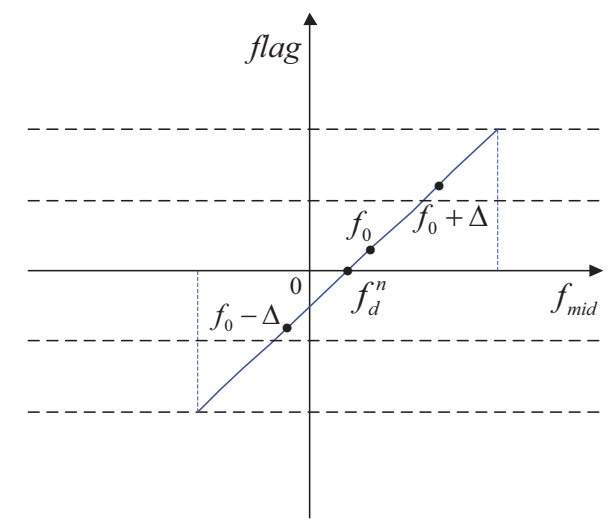

Fig. 7. Schematic diagram of bisection algorithm 


\section{Frequency offset linear interpolation and compensation}

As mentioned earlier since the UWA channel is affected by many factors, the channel parameters may vary quickly within a frame, especially when there is a high moving speed between the transmitter and receiver. Therefore the Doppler may shift greatly during a frame period. And this will unavoidably degrade the decoding performance. In the system, the data part of each received data frame is divided into $\mathrm{K}$ blocks as show in Fig. 8. Channel estimation and turbo equalization will be performed block by block iteratively to track the change of the channel. The same idea is proposed in CFO estimation and compensation.

\begin{tabular}{|c|c|c|c|c|c|c|c|}
\hline & $f_{d}^{n-1}$ & $f_{n}^{1}$ & $f_{n}^{2}$ & & $f_{n}^{K}$ & $f_{d}^{n}$ & \\
\hline$\cdots$ & Training & $\begin{array}{c}\text { Block } \\
1\end{array}$ & $\begin{array}{c}\text { Block } \\
2\end{array}$ & $\ldots$ & $\begin{array}{c}\text { Block } \\
\mathrm{K}\end{array}$ & Training & $\cdots$ \\
\hline
\end{tabular}

Fig. 8. Frame blocks of CFO linear interpolition

As shown in the Fig. 8, the Doppler shift of each data block is calculated with the CFO of previous frame $f_{d}^{n-1}$ and current frame $f_{d}^{n}$ by linear interpolation. The Doppler of the $i$-th block can be expressed as

$$
f_{n}^{i}=f_{d}^{n-1}+\frac{i\left(f_{d}^{n}-f_{d}^{n-1}\right)}{K+1}
$$

where $i=1,2 \cdots K$. Finally the received data will be resampled by the Doppler value of each data block to compensate the CFO.

\section{EXPERIMENT ARRANGEMENT}

To verify the effectiveness and performance of the system including the proposed CFO estimation and compensation algorithm in this paper, a UWA communication experiment was conducted in December 2012 in the Indian Ocean off Rottnest Island, Western Australia. The following is the detailed experimental scenario.

As shown in Fig. 9, the receiver (recorder) was fixed on the sea bed close to the Rottnest Waverider Buoy. The red dots with labels of T52, T54, T55, T56, T57, T58, T59, T60, and T61 denote different transmitter positions range from $125 \mathrm{~m}$ to $10 \mathrm{~km}$ from the receiver. The average water depth is $50 \mathrm{~m}$.

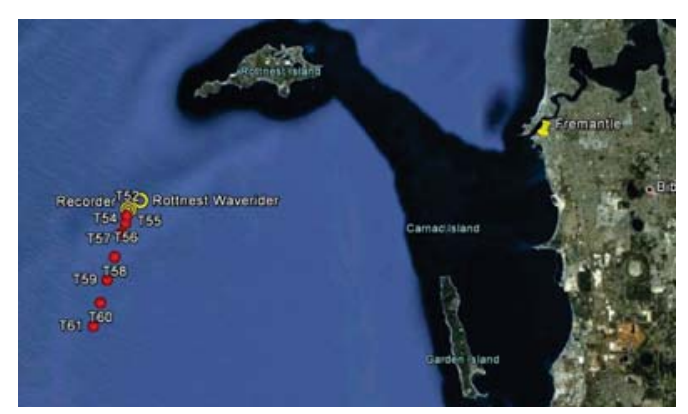

Fig. 9. Location of the experiment environment

Fig.10 shows the arrangement of transmitter and receiver. At the transmitter, a single transducer at $20 \mathrm{~m}$ depth below the

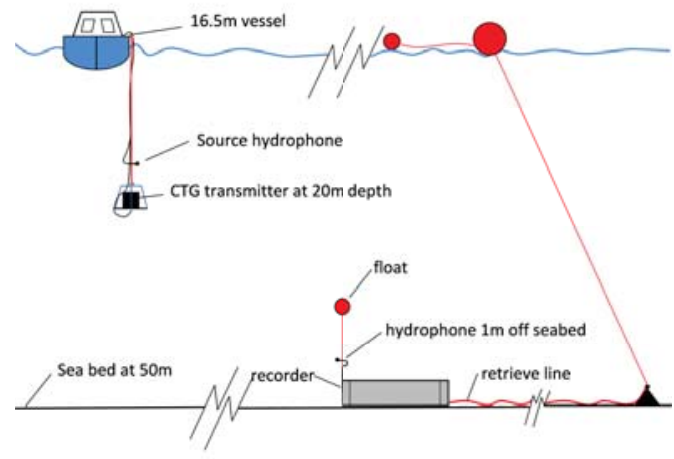

Fig. 10. Arrangement of transmitter and rrceiver

water was attached to a drifting vessel by a cable. At the $1 \mathrm{~km}$ range according to the GPS data, the average drift speed of the vessel was $0.96 \mathrm{~m} / \mathrm{s}$, and the peak drift speed is $1.7 \mathrm{~m} / \mathrm{s}$. At the receiver, a single hydrophone was attached by a cable at $1 \mathrm{~m}$ above the seabed. The drifting transmitter was set to be flexible to explore different communication ranges, which also allows movement of the transducer and hydrophone. This arrangement increased the Doppler shifts and Doppler spreading, and made channel estimation and tracking more challenging.

Signals were modulated to $12 \mathrm{kHz}$ center frequency for transmission by the transmit transducer with $4 \mathrm{kHz}$ system bandwidth. Transmitted and received signals were sampled by $96 \mathrm{kHz}$ sampling rate. 8PSK and QPSK modulations were chosen at ranges of $125 \mathrm{~m}, 250 \mathrm{~m}, 500 \mathrm{~m}, 1 \mathrm{~km}, 2 \mathrm{~km}$, and 4 $\mathrm{km}$, while QPSK and BPSK were used at the ranges of $6 \mathrm{~km}$ and $8 \mathrm{~km}$. At the range of $10 \mathrm{~km}$, only BPSK was adopted.

\section{EXPERIMENT RESULTS}

Fig. 11 shows the results of CFO estimation of consecutive 15 frames in the experiment at $1 \mathrm{~km}$ range with QPSK and $10 \mathrm{~km}$ range with BPSK. It can be observed that there is still a gap between the coarse and fine estimation results. During the experiment we noted that although the gap seems small but can result in remarkable impact on the performance because of the transitivity of Doppler shift in the sampling process, as shown in Fig. 12 and Fig .13. The Doppler at $1 \mathrm{~km}$ was relatively small and wandered around the X-axis while the Doppler at $10 \mathrm{~km}$ was basically below the $\mathrm{X}$-axis which illustrated a larger mobile speed of the transmitter. The irregular shape of the curve also illustrates the random shift of Doppler between adjacent frames.

Fig. 12 shows the comparison of BER at the $1 \mathrm{~km}$ and $10 \mathrm{~km}$ range of consecutive 15 frames with and without CFO linear interpolation, and comparison of BER with and without fine CFO estimation (with linear interpolation). We notice that when the channel condition is relatively bad and the Doppler varies fast within a frame, the algorithm with linear interpolation performs better apparently than the one without interpolation. When the channel environment is better the advantage is relatively small. And the result also illustrates the necessity of fine CFO estimation.

Table. 1 shows the average BER of consecutive 100 frames over the range of different distances from $1 \mathrm{~km}$ to $10 \mathrm{~km}$ before 


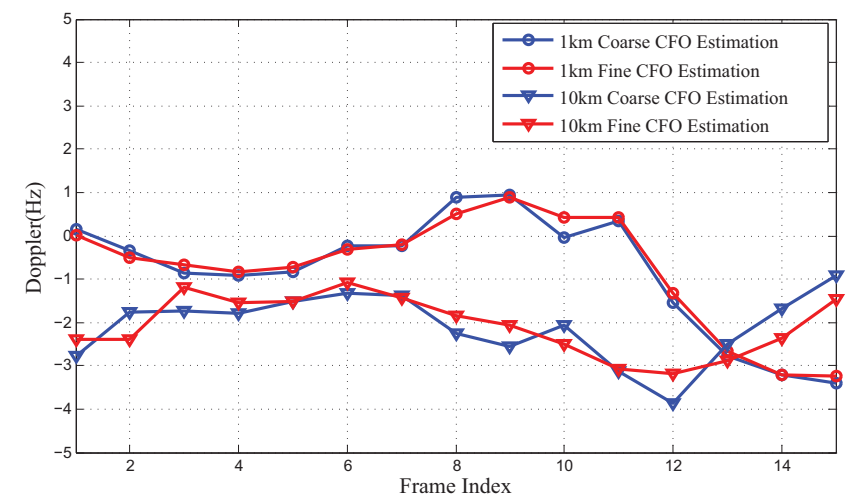

Fig. 11. CFO estimation results at $1 \mathrm{~km}$ and $10 \mathrm{~km}$

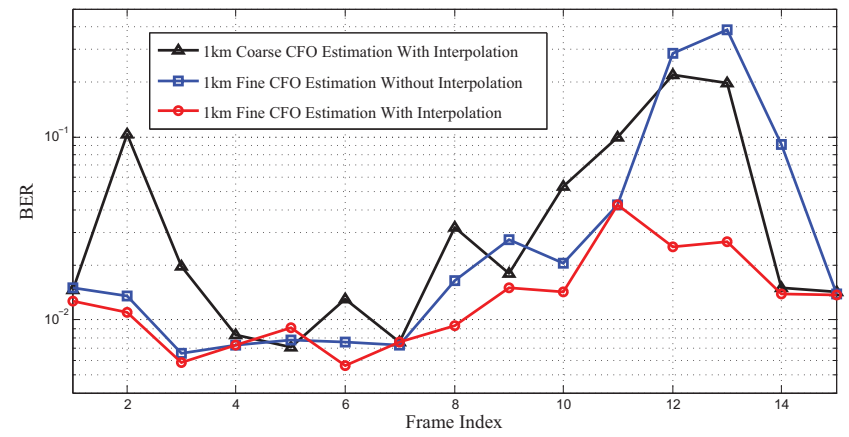

Fig. 12. Comparison of BER at the $1 \mathrm{~km}$ range

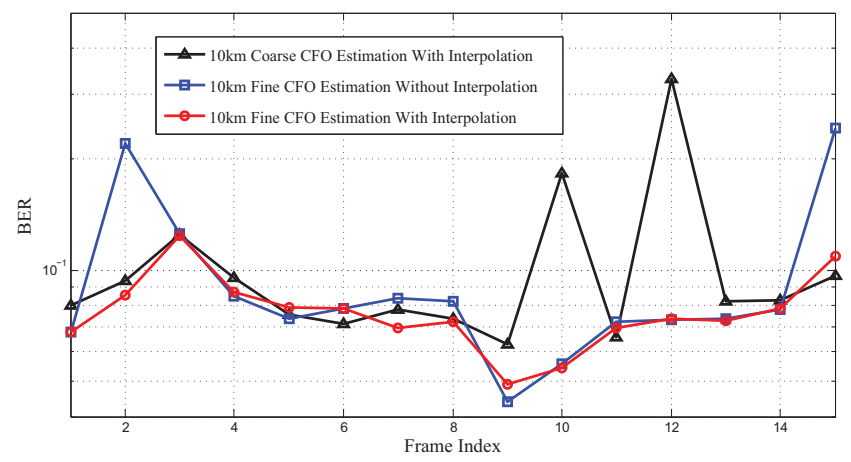

Fig. 13. Comparison of BER at the $10 \mathrm{~km}$ range

channel decoding. With the help of the proposed algorithm in this paper, the receiver can extract the data of all distances listed below. After the channel decoding the BER becomes zero. Generally the farther the distance is, the higher the BER will be. However we notice that since the channel environment at $2 \mathrm{~km}$ and $8 \mathrm{~km}$ is relatively bad, the BER is higher than supposed. But the system still performs well overall.

\section{CONCLUSION}

The result of $\mathrm{CFO}$ estimation and compensation has a significant impact on the decoding performance of the UWA system. In this paper, we propose a three-step scheme on a UWA communication system based on SC-FDE. First the coarse CFO estimation gives a rough Doppler value at the right offset range. Then the fine CFO estimation calculates a
TABLE I. BER of Different Distances Before CHANNEL DECODING

\begin{tabular}{|c|c|c|c|}
\hline Distance & $1 \mathrm{~km}$ & $2 \mathrm{~km}$ & $4 \mathrm{~km}$ \\
\hline Demodulation & QPSK & QPSK & QPSK \\
\hline BER & $1.95 \%$ & $12.16 \%$ & $2.98 \%$ \\
\hline \multicolumn{4}{|l}{} \\
\hline Distance & $6 \mathrm{~km}$ & $8 \mathrm{~km}$ & $10 \mathrm{~km}$ \\
\hline Demodulation & BPSK & BPSK & BPSK \\
\hline BER & $2.96 \%$ & $8.42 \%$ & $5.57 \%$ \\
\hline
\end{tabular}

more precise result using bisection method based on the rough value. Finally the whole received samples are compensated by resampling block by block with the CFOs obtained from linear interpolation. The scheme can adapt different volatile UWA channel environment in high speed scenario by adjusting the corresponding processing parameters. Results of the experiment shown the accuracy and good performance of the proposed algorithm. In fact, the scheme can contribute to extract the received data at all distance ranges in the experiment and achieve a $1.95 \%$ uncoded BER over a range of $1 \mathrm{~km}$ and $5.57 \%$ at $10 \mathrm{~km}$.

\section{REFERENCES}

[1] D. Kilfoyle and A. Baggeroer, "The state of the art in underwater acoustic telemetry," IEEE J. Oceanic Eng., vol. 25, pp. 4-27, Jan. 2000.

[2] A. Radosevic, J. G. Proakis, and M. Stojanovic, "Statistical characterization and capacity of shallow water acoustic channels," in Proc.IEEE OCEANS Conf., Bremen, Germany, 2009.

[3] M. Stojanovic and J. Preisig, "Underwater acoustic communication channels: Propagation models and statistical characterization," IEEE Communications Magazine, vol. 47, pp. 84-89, Jan. 2009.

[4] J. S. Dhanoa, R. F. Ormondroyd, "Combined Differential Doppler and Time Delay Compensation for an Underwater Acoustic Communication System,' in OCEANS '02 MTS/IEEE, Biloxi, USA, 2002.

[5] B. S. Sharif, J. Neasham, O. R. Hinton, A. E. Adams, "A computationally efficient Doppler compensation system for underwater acoustic communications," Oceanic Engineering, IEEE Journal of, vol.25, pp.52-61, Jan. 2000.

[6] T. J. Yang and L. Hu, "An improved frequency offset estimation algorithm for OFDM system," in Information Networking and Automation (ICINA), Kunming, China, 2010.

[7] K. Taehyuk and R. A. Iltis, "Iterative carrier frequency offset and channel estimation for underwater acoustic OFDM systems," Selected Areas in Communications, IEEE Journal, vol. 26, pp. 1650-1661, Dec. 2008.

[8] J. Trubuil, T. Le Gall and T. Chonavel, "Synchronization, Doppler and channel estimation for OFDM underwater acoustic communications," in OCEANS 2014-TAIPEI. IEEE, Taipei, Taiwan, 2014.

[9] X. L. Cheng, F. Z. Qu, and L. Q. Yang, "Single carrier FDMA over underwater acoustic channels," in Communications and Networking in China (CHINACOM), 2011 6th International ICST Conference on. IEEE, Harbin, China, 2011.

[10] X. H. Zhang, E. Q. Chen, and X. M. Mu, "Single-Carrier FrequencyDomain Equalization Based on Frequency-Domain Oversampling," IEEE communications letters, vol. 16. pp. 24-26, Dec. 2012.

[11] S. C. Guo, Z. Q. He, W. P. Jiang, et al, "Channel estimation based on compressed sensing in high-speed underwater acoustic communication," in Information, Communications and Signal Processing (ICICS) 2013, Tainan, Taiwan, 2013.

[12] Y. B. Ou, Z. Q. He, W. P. Jiang, et al, " Single-carrier iterative frequency-domain equalization with soft decision feedback in shallow underwater acoustic communication," in Information, Communications and Signal Processing (ICICS) 2013, Tainan, Taiwan, 2013. 